\title{
Signaling Capacity of FR4 PCB Traces for Chip-to-Chip Communication
}

\author{
Marcus van Ierssel, Tooraj Esmailian, Ali Sheikholeslami, and Pas S. Pasupathy \\ Dept. of Elec. and Comp. Eng., University of Toronto, \\ 10 King's College Rd., Toronto, Canada M5S 3G4 \\ Email: \{vane@eecg, tooraj@eecg, ali@eecg,pas@comm\}.utoronto.ca
}

\begin{abstract}
The signaling capacity of traces on the popular PCB dielectric material FR4 is under-utilized today by at least one order of magnitude through the choice of pre-coding, pulseshaping, equalization, and receiver architecture. This paper determines the channel capacity of FR4 traces of various length in the presence of noise and crosstalk, and explores capacity limits based on signaling schemes such as 4-level PAM.
\end{abstract}

\section{INTRODUCTION}

Signaling rates between chips on printed circuit boards (PCB) have increased rapidly in recent years. $3.6 \mathrm{~Gb} / \mathrm{s} / \mathrm{pin}$ and $5 \mathrm{~Gb} / \mathrm{s} /$ pin implementations have been reported in [1] and [2], respectively, using basic communication techniques such as pulse shaping in the form of slew-rate control. Higher signaling rates can be achieved at the expense of more sophisticated signaling techniques. This paper examines the limits of signaling on $\mathrm{PCB}$ traces using Shannon's capacity theorem and the waterpouring method [3] using realistic assumptions of noise and crosstalk in such systems and physical characteristics of the traces [4] for the dielectric material FR4. Section 3 shows that this capacity exceeds $100 \mathrm{~Gb} / \mathrm{s}$ for a $25 \mathrm{~cm}$-long trace using a $100 \mathrm{miV}$ transmitted signal. Section 4 shows how this capacity is under-utilized in today's implementations and offers insight into areas where more efficient techniques can be used.

\section{CHANNEL CAPACITY}

The channel capacity defines an upper bound on the information rate that can be reliably transmitted through the channel, a PCB trace in this case. This rate is a function of three parameters in the system model, as illustrated in Figure 1: The channel transfer function $H(f)$, the Gaussian noise spectral power density (SPD) $S_{n}(f)$, and the SPD of the transmitted signal $S_{x}(f)$.

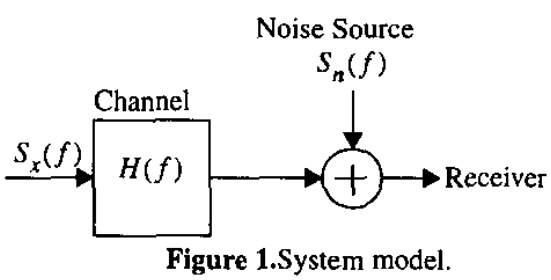

If we assume $H(f)$ and $S_{n}(f)$ are fixed, and limit the average transmit power to $E\left[x^{2}(t)\right]=P_{S}=\int_{0}^{\infty} S_{x}(f) d f$, there are an infinite number of possible input power spectral densities, each resulting in different capacities. Finding the transmit PSD that maximizes the capacity given a fixed total input power requires the application of the waterpouring method [5]. To calculate the channel capacity based on the water-pouring method using (1)-(3) we first calculate $S_{n}(f) /|H(f)|^{2}$. The channel capacity can then be calculated as

$$
C=\int_{f \in F_{L}} \log \left[1+\frac{S_{x}(f)|H(f)|^{2}}{S_{n}(f)}\right] d f \text { bits/s, }
$$

where $F_{L}$ is the frequency band for which $S_{n}(f) /|H(f)|^{2} \leq L$, and $L$ is the solution to

$$
P_{S}=\int_{f \in F_{L}}\left[L-\frac{S_{n}(f)}{|H(f)|^{2}}\right] d f
$$

where $S_{x}(f)$ can be computed as

$$
S_{x}(f)=\left\{\begin{array}{c}
L-\frac{S_{n}(f)}{|H(f)|^{2}} ; f \in F_{L} \\
0 ; f \notin F_{L}
\end{array}\right.
$$

Solving (1)-(3) requires the evaluation of $H(f)$ and $S_{n}(f)$. 


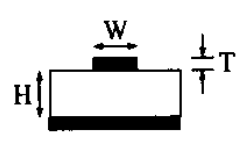

(a)

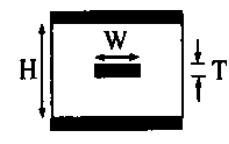

(b)
Figure 2.Cross-section of (a) microstrip line (b)

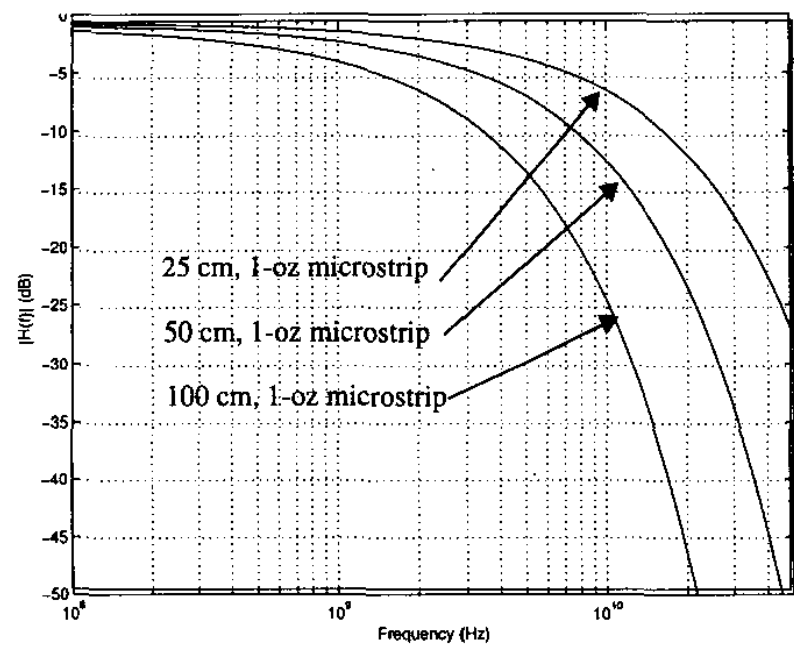

Figure 3.Transfer function magnitude of 1-oz, FR4 PCB traces (microstrip). $\mathrm{Z}_{\mathrm{c}}=56 \Omega, \mathrm{H}=.006$ ", $\mathrm{W}=.008^{\prime \prime}, \mathrm{T}=.00137^{\prime \prime}, \varepsilon_{\mathrm{r}}=$ 4.5 and $\tan \delta_{1}=0.025$.

We have computed $H(f)$ using Matlab (verified using Hspice) for various-length FR4 PCB traces using per-unitlength parameters of $1-\mathrm{oz}$ microstrip line; A microstrip is a trace (wire) on the surface of a PCB, having only one reference plane, as shown in Figure 2. A PCB trace sandwiched between two reference planes is defined as a stripline, and produces similar results for $H(f)$. Striplines, however, do not suffer from far-end crosstalk, an important property that will be shown to be beneficial to channel capacity.

The simulated channel transfer functions $H(f)$ are shown in Figure 3 and indicate that current signaling rates are already exceeding the bandwidth of longer traces. These bandwidth limitations motivate the investigation of the channel capacity.

Next, we determine $S_{n}(f)$. There are two dominant sources that contribute to the total noise: thermal noise and shot noise. First, thermal noise is modeled as a white Gaussian noise source with an RMS voltage of

$$
V_{T h}=\sqrt{4 k_{B} T R B},
$$

in which $k_{B}$ is a constant $\left(1.38 \times 10^{-23} \mathrm{~J} / \mathrm{K}\right), T$ is the temperature in Kelvin, $R$ is resistance in Ohms, and $B$ is the bandwidth in Hertz. Over a bandwidth of $10 \mathrm{GHz}$ and for a receiver impedance of $50 \Omega$, we have $V_{T h}=91 \mu \mathrm{V}$. If $P$ represents the noise power:

$$
V_{T h}=\sqrt{P \times R}=\sqrt{2 \int_{0}^{B} S_{n}(f) d f \times 50} .
$$

Assuming white thermal noise: $S_{n}(f)=8.21 \times 10^{-21} \mathrm{~W} / \mathrm{Hz}$. Second, shot noise is modeled as a white Gaussian noise source with an RMS current of

$$
I_{S h}=\sqrt{2 q I B}
$$

in which $q$ is the charge of an electron $\left(1.6 \times 10^{-19} \mathrm{C}\right), I$ is the current in Amps, and $B$ is the system bandwidth in Hertz. For a typical value of $10 \mathrm{~mA}$ of current and over $10 \mathrm{GHz}$ of bandwidth the shot noise is $5.66 \mu \mathrm{A}$, which induces $283 \mu \mathrm{V}$ across a $50 \Omega$ impedance. Therefore, assuming white shot noise $S_{n}(f)=7.96 \times 10^{-20} \mathrm{~W} / \mathrm{Hz}$.

We now use $H(f)$ and $S_{n}(f)$ as derived above to calculate the channel capacity for a number of PCB traces with different lengths.

\section{EVALUATION OF CAPACITY LIMITS}

This section investigates the channel capacity of traces with and without the influence of crosstalk from neighbouring traces.

\subsection{CROSSTALK-FREE CAPACITY}

Crosstalk-free capacity exists when the noise in a channel is independent of signals in other channels. Some examples for crosstalk-free environments are when there is only one line carrying a high speed communication signal, or when parallel striplines are signaling in the same direction. Figure 4 . shows the capacity of different length 1-oz microstrip lines versus RMS voltage of the transmitted signal. This figure shows the channel capacity to be more than an order of magnitude greater than the bit rate used in current $\mathrm{PCB}$ signaling schemes. Note that the lower transmit voltages would likely require a lower current, and hence result in lower shot noise, making Figure 4 somewhat conservative. The computed capacity for a stripline with parameters similar to a microstrip shows similar results.

These capacity curves have been computed using the waterpouring method and assume that the transmit power spectral density, $S_{x}(f)$, in each case is chosen in a way that maximizes the channel capacity. The bandwidth required by $s_{x}(f)$ is shown in Figure 5. Note that the longer traces require less bandwidth for the same capacity due to the inefficiency of putting signal power into the highly attenuated higher frequencies. 


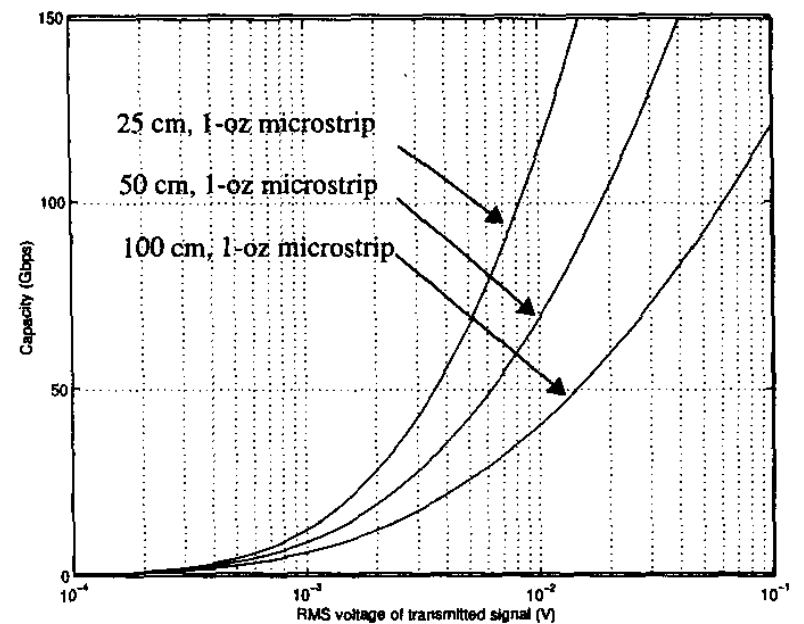

Figure 4.Crosstalk free capacity of 1-oz microstrip traces with different length versus the RMS voltage of transmitted signal. The parameters of traces are the same as those of Figure 3.

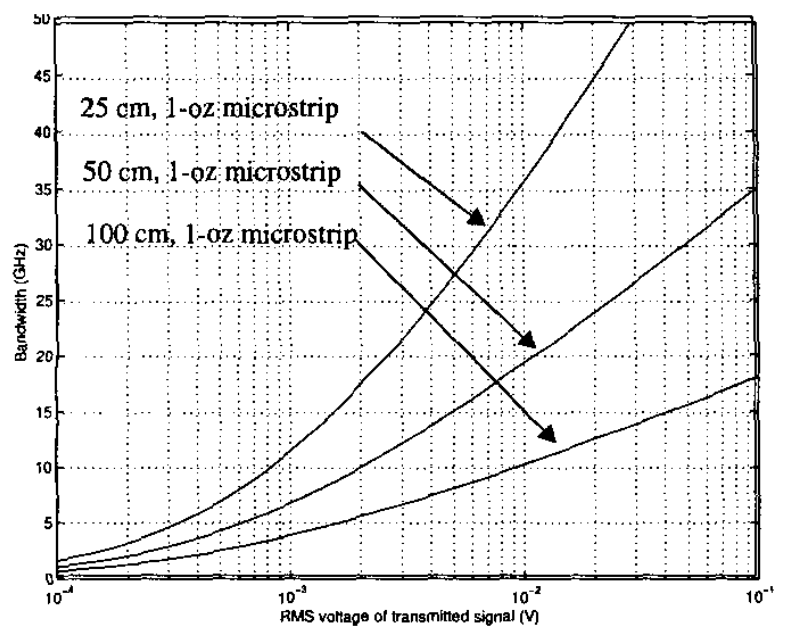

Figure 5.Bandwidth required to achieve capacity shown in Figure 4.

\subsection{CAPACITY IN PRESENCE OF CROSSTALK}

When crosstalk is the dominant noise in the system, which is often the case in microstrip lines, the channel capacity is independent of transmit PSD, $S_{x}(f)$, and is given by [6]:

$$
C_{\text {cross }}=\int_{f \in F} \log _{2}\left[1+\left|\frac{H_{\text {chan }}(f)}{H_{\text {cross }}(f)}\right|^{2}\right] d f \text { bits } / \mathrm{s},
$$

in which $H_{c h a n}(f)$ and $H_{c r o s s}(f)$ are channel and crosstalk frequency responses and $F$ is the frequency range in which power is transmitted. Figure 6 shows the crosstalk-limited channel capacities for different channel lengths. Note that since the capacity is independent of $S_{x}(f)$, the x-axis is

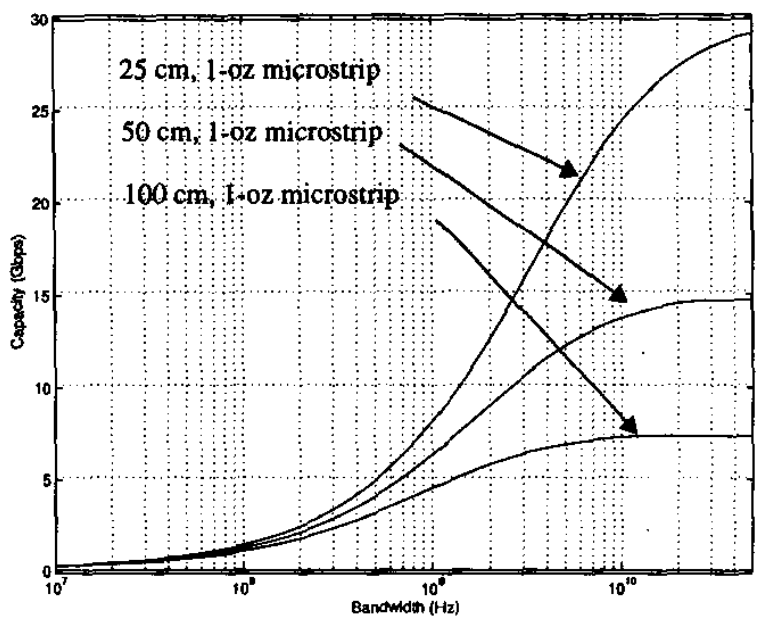

Figure 6.Capacity of 1-oz microstrip traces with different length versus the utilized bandwidth, when channel is crosstalk limited. The parameters of traces are the same as those of traces in Figure 3.

bandwidth, not transmit RMS voltage. Figure 6 shows that the capacity of microstrips approaches a limit, regardless of the bandwidth or transmit voltage. This demonstrates the necessity of using striplines for long PCB traces in a crosstalk environment since they are immune to far-end crosstalk.

\section{PAM SIGNALING}

The channel capacities derived up to this point require a complex signaling scheme, resulting in a complex transceiver implementation. The practical limitations in high-speed signaling dictate a simple implementation such as pulse amplitude modulation (PAM), typically with 2,4 , or 8 levels. It is also useful to find the capacity limits of this method of signaling.

\begin{tabular}{l|l}
\hline BER & MTBF (@10 Gbps) \\
\hline $10^{-14}$ & 2.8 hours \\
\hline $10^{-16}$ & 11.6 days \\
\hline $10^{-18}$ & 3.2 years \\
\hline
\end{tabular}

Table 1: BER and equivalent MTBF.

Uncoded PAM signaling will always be subject to some finite bit error rate (BER), so the capacity must be specified at a particular BER. Table 1 lists the mean time between failure (MTBF) for various BERs at 10 Gbps. A BER of $10^{-18}$ is targeted since BERs greater than $10^{-18}$ result in an unacceptably low MTBF. The symbol error rate for a PAM signaling scheme is computed as [3]: 


$$
P_{e} \approx K \cdot Q\left(\frac{d_{\min }}{2 \sigma}\right)
$$

in which $K$ is a constant related to the PAM constellation size; $d_{\min }$ is the minimum distance between the points of the PAM constellation, and $\sigma$ is the standard deviation of the received noise. If a Gray coding scheme is used then the bit error rate is approximately $P_{e} / \log _{2} M$ for M-PAM signaling.

Using (7), we now determine the minimum RMS voltage required for a PAM signal to achieve the desired BER. We derive the case for the $100 \%$ excess bandwidth raised cosine pulse in a $10 \mathrm{GHz}$ bandwidth on a $25 \mathrm{~cm}$ microstrip line. This result is presented in Table 2 . These results are also shown in Figure 7 where they are contrasted with the channel capacity. As can be seen, there is a large gap between the capacity and what PAM signaling can achieve. For example, with an RMS voltage of approximately $12 \mathrm{mV}$ for 8-PAM signaling at a BER of $10^{-18}$ a bit rate of $30 \mathrm{Gbps}$ is achievable, while the channel capacity is close to $200 \mathrm{Gbps}$.

\begin{tabular}{l||lcc}
\hline \multicolumn{1}{c||}{} & \multicolumn{3}{c}{ RMS Transmit Voltage (mV) } \\
BER & 2-PAM & 4-PAM & 8-PAM \\
\hline $10^{-14}$ & 2.40 & 5.35 & 10.92 \\
\hline $10^{-16}$ & 2.58 & 5.77 & 11.75 \\
\hline $10^{-18}$ & 2.65 & 6.13 & 12.52 \\
\hline
\end{tabular}

Table 2: The required RMS voltage at the transmitter for a $25 \mathrm{~cm}$ microstrip in order to achieve different BERs at the receiver with different sizes of PAM constellation.

While the signaling rates achievable with PAM are shown to be less than the channel capacity, the signaling rates achieved in [1] and [2] are even lower. This indicates that with circuit improvements and the application of additional communication techniques, such as equalization and coding, it is possible to further increase the signaling rates on FR4.

\section{CONCLUSIONS}

PCB traces implemented on FR4 material have a fundamental capacity limit much higher than what current transceivers exploit. In a crosstalk environment far-end crosstalk reduces the capacity of microstrips, requiring the use of striplines. In order to get close to the channel capacity multi-carrier modulation and error control coding techniques are necessary. PAM signaling is a simple modulation technique, used because of its

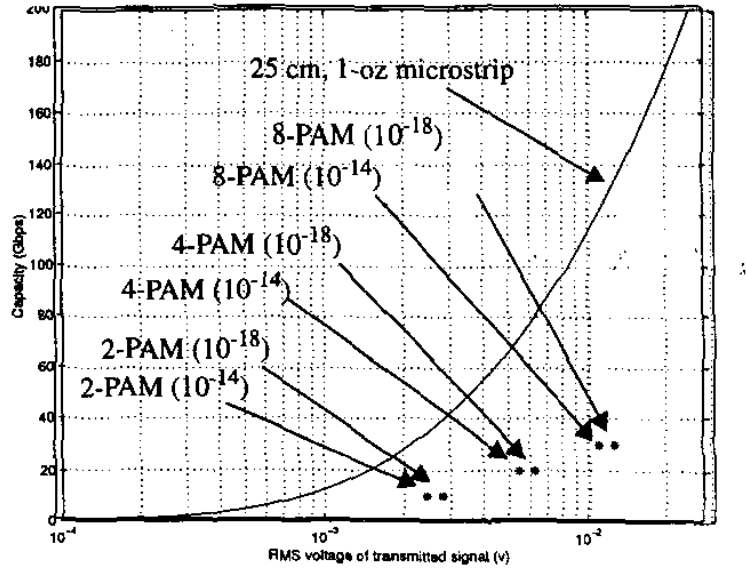

Figure 7. Capacity of $1-\mathrm{oz}, 25 \mathrm{~cm}$ microstrip traces versus the RMS voltage of transmitted signal in $10 \mathrm{GHz}$ bandwidth and operating point of different PAM sizes at different BER.

ease of implementation, that results in a maximum bitrate less than the wire's capacity. However, the maximum bitrate achievable for PAM signaling exceeds the bit rate demonstrated in current implementations, implying further increases are possible for FR4 signaling rates.

\section{ACKNOWLEDGMENT}

The authors would like to acknowledge the generous funding of Fujitsu Labs of America and NSERC of Canada.

\section{REFERENCES}

[1] Matthew Haycock, and Randy Mooney, "3.2GHz 6.4G $\mathrm{G} / \mathrm{s}$ per Wire Signaling in $0.18 \mu \mathrm{m}$ CMOS", IEEE Int. Solid-State Circuits Conf.(ISSCC) Dig. Tech. Papers, pp. 62-63, 2001.

[2] Hirotaka Tamura, et. al, " $5 \mathrm{~Gb} / \mathrm{s}$ Bidirectional BalancedLine Link Compliant with Plesiochronous Clocking", IEEE Int. Solid-State Circuits Conf.(ISSCC) Dig. Tech. Papers, pp. 64-65, 2001.

[3] E. Lee and D. Messerschmitt, Digital Communication, Kluwer, 1994.

[4] A. Deutsch, G. V. Kopcsay, et. al," "Frequency-Dependent Losses on High-Performance Interconnections," IEEE Trans. on Electromagnetic Compatibility, pp. 446-465, Vol. 43, No. 4, Nov. 2001.

[5] R. Gallager, Information Theory \& Reliable Communication, Wiley, 1968.

[6] I. Kalet, and S. Shamai, "On the Capacity of Twisted-Wire Pair: Gaussian Model," IEEE Trans. on Commun., pp. 379383, Vol. 38, No. 3, March 1990. 